\title{
Correction: Precision Public Health Campaign: Delivering Persuasive Messages to Relevant Segments Through Targeted Advertisements on Social Media
}

\author{
Jisun $\mathrm{An}^{1}$, PhD; Haewoon Kwak ${ }^{1}, \mathrm{PhD}$; Hanya M Qureshi ${ }^{2}$, BA; Ingmar Weber ${ }^{3}, \mathrm{PhD}$
}

\footnotetext{
${ }^{1}$ School of Computing and Information Systems, Singapore Management University, Singapore, Singapore

${ }^{2}$ Yale School of Medicine, Yale University, New Haven, CT, United States

${ }^{3}$ Qatar Computing Research Institute, Hamad Bin Khalifa University, Doha, Qatar
}

\section{Corresponding Author:}

Jisun An, PhD

School of Computing and Information Systems

Singapore Management University

80 Stamford Road

Singapore, 178902

Singapore

Phone: 6568264809

Email: an.jisun.221@gmail.com

\section{Related Article:}

Correction of: http://formative.jmir.org/2021/9/e22313/

(JMIR Form Res 2021;5(10):e33922) doi: 10.2196/33922

In "Precision Public Health Campaign: Delivering Persuasive Messages to Relevant Segments Through Targeted Advertisements on Social Media" (JMIR Form Res 2021;5(9):e22313) the authors noted one error.

In the originally published manuscript, the order of authors Ingmar Weber and Hanya M Qureshi was reversed. This has been corrected to reflect that Hanya M Qureshi is the paper's third author and Ingmar Weber is the paper's fourth author. The author affiliations have been renumbered accordingly.

The full list of authorship and affiliations in the originally published version appeared as follows:

Jisun $A n^{l}, P h D ;$ Haewoon Kwak ${ }^{l}, P h D$; Ingmar Weber $^{2}$, PhD; Hanya M Qureshi ${ }^{3}, B A$

${ }^{1}$ School of Computing and Information Systems, Singapore Management University, Singapore, Singapore

${ }^{2}$ Qatar Computing Research Institute, Hamad Bin Khalifa University, Doha, Qatar

\author{
${ }^{3}$ Yale School of Medicine, Yale University, New \\ Haven, CT, United States
}

The full list of authorship and affiliations has been updated as follows in the corrected version:

Jisun $\mathrm{An}^{1}$, PhD; Haewoon Kwak ${ }^{1}$, PhD; Hanya M
Qureshi ${ }^{2}, \mathrm{BA}$; Ingmar Weber ${ }^{3}, \mathrm{PhD}$
${ }^{1}$ School of Computing and Information Systems,
Singapore Management University, Singapore,
Singapore
${ }^{2}$ Yale School of Medicine, Yale University, New
Haven, CT, United States
${ }^{3}$ Qatar Computing Research Institute, Hamad Bin
Khalifa University, Doha, Qatar

The correction will appear in the online version of the paper on the JMIR Publications website on October 5, 2021, together with the publication of this correction notice. Because this was made after submission to PubMed, PubMed Central, and other full-text repositories, the corrected article has also been resubmitted to those repositories. 
This is a non-peer-reviewed article. Submitted 29.09.21; accepted 29.09.21; published 05.10.21.

Please cite as:

An J, Kwak H, Qureshi HM, Weber I

Correction: Precision Public Health Campaign: Delivering Persuasive Messages to Relevant Segments Through Targeted Advertisements on Social Media

JMIR Form Res 2021;5(10):e33922

URL: https://formative.jmir.org/2021/10/e33922

doi: $10.2196 / 33922$

PMID:

(CJisun An, Haewoon Kwak, Hanya M Qureshi, Ingmar Weber. Originally published in JMIR Formative Research (https://formative.jmir.org), 05.10.2021. This is an open-access article distributed under the terms of the Creative Commons Attribution License (https://creativecommons.org/licenses/by/4.0/), which permits unrestricted use, distribution, and reproduction in any medium, provided the original work, first published in JMIR Formative Research, is properly cited. The complete bibliographic information, a link to the original publication on https://formative.jmir.org, as well as this copyright and license information must be included. 